\title{
Chiara Xausa \\ «Nello schedario del mio cuore»: i romanzi di memoria di Giacoma Limentani
}

\begin{abstract}
Il contributo si propone di leggere la Trilogia di Giacoma Limentani come risposta a una memoria della Shoah tendente a omologare in un'oppressione condivisa le diverse testimonianze, in particolare quelle delle donne. I suoi romanzi saranno analizzati a partire dalle riflessioni sul significato e sulla funzione della memoria che li attraversano: In contumacia, Dentro la $\mathrm{D}$ e La spirale della tigre sono infatti un viaggio dal ricordo che opprime a quello che salva e permette di guardare al futuro con speranza. In contumacia nasce quando la narrazione dei ricordi di infanzia diventa un atto di sopravvivenza e una necessità; procede per frammenti, facendosi testimone del portato emotivo del trauma. In Dentro la $D$ il recupero dell'eredità familiare e dell'identità ebraica si fanno dono e le aprono una porta verso la dignità, che, quando la distanza temporale ha permesso di storicizzare il dolore, la guida nella scrittura de La spirale della tigre. Qui spirali di memorie intrecciano storie personali a quelle dei personaggi e delle personagge della tribù del ghetto di Roma: è narrando la particolarità delle loro vite e recuperando le memorie infrante dalla Shoah che il passato smette di proiettare ombre sul futuro e la memoria privata diventa collettiva, senza tuttavia essere omologante.
\end{abstract}

\section{Memorie marginali}

Nel 1994 si tiene a Torino il primo convegno sulla deportazione delle donne italiane, promosso dall'Associazione Nazionale ex Deportati (Aned). È l'inizio di una ricerca nazionale sul tema, in un paese in cui le memorie di donne pubblicate nel dopoguerra sono appena una ventina su centocinquantanove testimonianze (BrAvo 1995). Una tale disparità difficilmente è riconducibile al minor numero di deportate e di sopravvissute, spiegazione che ha prevalso per lungo tempo negli studi sulla Shoah. Tra le giustificazioni addotte, quella che ricorre con più frequenza è l'indicibilità: non disponendo di parole per descrivere l'orrore consumatosi ad Auschwitz, pochissime donne riescono a raccontarlo. Tuttavia, l’indicibilità è anche la causa di molti dei silenzi maschili, e da sola non spiega quelli delle donne, che si trovano a dover utilizzare un linguaggio nel quale la loro

The e-journal «altrelettere» is hosted at the URL: http://www.altrelettere.uzh.ch , in accordance with the Open Access Policy of the University of Zurich. Please cite this article as follows: Chiara XAUSA, «Nello schedario del mio cuore»: i romanzi di memoria di Giacoma Limentani in «altrelettere», 1.07.2018, DOI: 10.5903/al_uzh-40.

(C) This article is licensed under a Creative Commons Attribution 2.5. Switzerland (CC BY-NC-ND 2.5). Please read the license terms on the website: http://creativecommons.org/licenses/by-nc-nd/2.5/ch/deed.en 
specifica condizione è resa invisibile: servirsene per scrivere di se stesse le porta a pensarsi nel modo in cui l'Altro, il neutro universale, le rappresenta. La memoria collettiva omologante della Shoah condiziona in tal modo le memorie individuali e silenzia quelle marginali. A volte si decide di non dare ascolto alle testimonianze delle donne nella convinzione che troppo si sia già detto a riguardo, e che gli eccessi di memoria siano nocivi per la memoria stessa.

Il timore più grande è che una separazione di genere rischi di indebolire la memoria della Shoah. Si arriva in tal modo al paradosso per cui si dimentica il nesso tra il lager e la violenza sessuale contro le donne. Come ricordano Bravo e Jalla, «non si tratta solo di un effetto implicito dell'organizzazione concentrazionaria, ma di un obiettivo perseguito, che rimanda alla matrice assolutamente antifemminile del nazismo»(BRAVO, JALLA 1986, 206), per la quale non solo il corpo della donna è degradato a funzione, privo di identità e soggettività, ma la femminilità diventa oggetto di tortura, e la donna ridotta a meno di corpo. La mortificazione della femminilità riguarda tutte le donne del lager: le più belle, che potevano essere selezionate per i bordelli, e tutte le altre, che servivano per le sperimentazioni scientifiche. Ricorda ancora Anna Bravo:

Essere prigioniere vuol dire dover esporre in pubblico, a sguardi di aguzzini, corpi abituati [...] a un pudore rigoroso; vedere quelli di altre, magari anziane, e restarne turbate; non potersi più riconoscere nella propria immagine fisica. Vuol dire vivere con bambini destinati a sparire, con compagne che arrivano incinte al Lager e si affannano per nutrire un figlio che verrà ucciso appena nato; scoprire nelle donne, anche in se stesse, una distruttività che non si sarebbe mai immaginata; subire, spinta all'estremo, una vita promiscua di cui non si ha alcuna esperienza, neppure quella che agli uomini viene dall'aver fatto il servizio militare e la guerra (BRAVO 1995, 19).

Gravidanze e parti, nelle condizioni estreme del lager, diventano pericolosi avvenimenti da nascondere, e assieme ai frequenti aborti, spontanei e indotti, e alle molestie sessuali, rendono le donne sempre più vulnerabili; anche l'amenorrea diventa una costante.

Nonostante la specificità della prigionia delle donne, la loro testimonianza è stata per lungo tempo appiattita e omologata in un'oppressione condivisa, e coperta nell'immediato dopoguerra da un pesante silenzio, dovuto anche al fatto 
che «per gli scritti femminili si usavano criteri di rilevanza ancora più selettivi di quelli applicati agli scritti di uomini - criteri di mercato e politico-ideologici piuttosto che culturali» (BRAvo 2009, 8). È nel clima culturale del femminismo degli anni 80 e 90 che iniziano a svilupparsi i primi filoni di studio sul rapporto tra la Shoah e il genere, provenienti inizialmente dalla storiografia americana e solo in anni seguenti da quella italiana. ${ }^{1}$

Risale ad anni più recenti il lavoro di Stefania Lucamante Quella difficile identità. Ebraismo e rappresentazioni letterarie della Shoah, che si sposta dall'ambito storiografico all'indagine sul testo letterario. Per proporre una lettura della Trilogia di Giacoma Limentani come risposta alla memoria omologante della Shoah - non solo della prigionia, ma anche di ciò che la precede -, si partirà dal capitolo di Lucamante dedicato a Rosetta Loy, Lia Levi e Giacoma Limentani, le «bambine di Roma». Loy, Levi e Limentani vivono la propria infanzia negli anni della promulgazione delle leggi razziali, e, nonostante la cattolica Loy non ne sia vittima, né le ebree Levi e Limentani vivano direttamente la deportazione, la loro identità di donne non potrà che essere costruita a partire dal trauma della Shoah. Se le generazioni che le precedono avevano visto l'apertura dei ghetti, integrazione e diversità scandiscono le vite delle bambine e dei bambini degli anni Trenta, che si chiedono se si possa ancora chiamare patria un paese per la cui Unità i genitori avevano combattuto e che ora vede l'ebreo come Altro. Giacoma Limentani, narrando i momenti in cui il padre veniva prelevato e poi riportato a casa dai «bravi» della vicina Casa del Fascio, che lo trascinavano e gettavano sul pavimento come un ubriaco, ricorda l'omertà dei condomini, e il loro conformismo mentale e culturale la conduce a una diversa modalità di pensare la testimonianza:

Restando ai miei occhi indispensabile ogni forma di onesta testimonianza, scritta oppure orale che sia, io credo che si dovrebbe cominciare a pretendere che non si provi orrore solo per il massimo, indicibile insulto della Shoah, ma di badare anche agli insulti minori che a quel massimo insulto hanno spianato la strada (LIMENTANI 2008, 227; corsivo di chi scrive). 
Riconoscere la particolarità delle testimonianze della Shoah contro un appiattimento dell'oppressione significa dunque non solo dare voce alla specificità della prigionia delle donne, ma anche a quella degli «insulti minori» antecedenti la deportazione. Significa rifiutare di identificarsi come testimone neutra, come se «dire io» fosse «incongruo di fronte alla natura frantumata e massificata dell'esperienza» (BRAVO, JALLA 1994, 63), e affermare invece la propria singolarità.

\section{Memoria come cura}

Nata nel 1927 a Roma, dove tutt'ora vive, Giacoma Limentani è una raffinata narratrice, saggista, traduttrice, e «maestra di sapienza ebraica», come la racconta Clara Sereni nella postfazione al Il gioco dei regni (SERENI 1993, 434). Tutta la sua produzione letteraria è attraversata da una continua riflessione sul significato e sulla funzione della memoria: non il semplice recupero del ricordo, ma una ricerca profonda, alle radici dell'anima, un interrogarsi che non pretende mai di trovare risposte definitive. I suoi romanzi di memorie, In contumacia, Dentro la $D$ e La spirale della tigre, ora accorpati insieme da Iacobelli con il titolo Trilogia, sono un viaggio dal ricordo che opprime a quello che salva, proiettandosi nel futuro. La scrittura permette di dimenticare e ricordare allo stesso tempo, guarire il dolore e storicizzarlo, comunicandolo attraverso la pagina letteraria.

Deve venire in aiuto l'età adulta perché Giacoma Limentani trovi la forza di tornare ai luoghi d'ombra di un'infanzia infranta, quando riesce a dare voce alla piccola Mina e rinascere a se stessa attraverso la scrittura. Più di vent'anni si rendono necessari per affrontare l'afasia e raccontare l'esperienza di bimba ed ebrea romana: la prima narrazione, In contumacia, intensamente autobiografica, viene data alle stampe nel 1967. Molto altro tempo passa poi prima di giungere alla serena accettazione dell'eredità familiare di Dentro la $D$ e al risarcimento simbolico ottenuto ne La spirale della tigre, pubblicati nel 1992 e nel 2003. Queste distanze temporali hanno poco a che vedere con l'impossibilità della 
rappresentazione letteraria dopo Auschwitz, soggetto «ipersfruttato» ma allo stesso tempo «ignorato» (LIMENTANI 1997, 9), perché, riflette Limentani, si scrive sempre «dopo»:

Scrivere, di qualsiasi argomento, per qualsiasi ragione e con qualsiasi finalità si scriva, è ed è sempre stato uno scrivere dopo. Dopo che si è imparato a scrivere, tanto per dirne una, e quindi dopo aver assunto un sia pur minimo bagaglio di nozioni, sentimenti, ricordi. Dopo aver avvertito la necessità di scrivere: per annotare quanto non va dimenticato, per rivelarsi a se stessi, per inserire la propria testimonianza nella scia di una tradizione amata, per comunicare con gli altri $(i v i, 19)$.

A impedirle di scrivere, negli anni immediatamente successivi al termine della guerra, non è stata solo la difficoltà di fare i conti con se stessa e di affrontare i traumi subiti, ma la paura di non trovare ascolto. L’indifferenza uccide, soffoca la parola. «L'indifferenza è l'aggressione» (LIMENTANI 2013, 15), si legge in apertura di In contumacia. L'apatia dimostrata dai condomini quando suo padre veniva gettato a terra e insultato come fosse un ubriaco, e il più generale disinteresse degli italiani nei confronti delle leggi per la difesa della razza, si tramutano in indifferenza per la testimonianza e ne paralizzano la scrittura.

Arriva però il giorno in cui la narrazione dei ricordi d'infanzia diventa un atto di sopravvivenza e una necessità, e solo la tessitura di una relazione con chi legge permette di guardare indietro a quei momenti che «non esistono, se nessuno li sa». In contumacia nasce in una nottata, scritto con la febbre alta, ripercorrendo le rovine dell'infanzia per ricostruire una soggettività scomposta. Scrivere diventa un fatto segnato da un'appartenenza di genere, in quanto legato all'esperienza biologica della gravidanza. Nella vocazione al romanzo agisce la

spinta di una fede particolarissima. [...] È grazie a una fede siffatta, in certo senso analoga alla fede nella nascita d'un figlio che si porti in grembo o, prima ancora, di cui si aneli il palpito nel proprio grembo, che si può trovare la forza di scrivere e, insieme, di vivere il conflitto fra ciò che si vuole scrivere, che preme per prendere forma $\mathrm{e}$ venire scritto, e il linguaggio che gli darà la forma adatta. [...] Io credo che per via di questo conflitto molte storie che pure premono per vedere la luce restano a lungo sospese fra pensiero e penna (LIMENTANI 2004a, 132).

Il rinascere e il dare la vita attraverso la narrazione sono immagini che troviamo in molta scrittura a firma di donne, ma che spesso, se legate a un evento 
traumatico, assumono un carattere grottesco. Si pensi a Edith Bruck: la scrittura è una «gravidanza infinita», il mostro-Auschwitz alberga nel suo grembo e lo devasta di giorno in giorno, senza alcuna possibilità di liberarsene. In Limentani, invece, una volta superato il conflitto tra quelle che lei definisce le due $P$, la storia a cui è stata donata la vita diventa un balsamo sia per chi l'ha scritta che per le lettrici e i lettori. In particolar modo quando dà forma a sradicamenti della memoria che chiedono con insistenza di materializzarsi nella pagina scritta.

Come Limentani lo scrive in una sola notte, noi sentiamo la necessità di affrontarlo tutto d'un fiato: impossibile respirare leggendo parole che si susseguono con una tale velocità. La scrittura di In contumacia non presenta alcuna narrazione dei fatti, ma un ininterrotto flusso di coscienza sull'evento drammatico e un continuo andirivieni tra presente e passato. Siamo a Roma, tra il 1938 e la Liberazione. La piccola Mina riceve la visita di quattro squadristi che sono alla ricerca del padre antifascista, quel giorno fortunatamente fuori casa. Se la bimba non rivela il nascondiglio del padre loro lo condannano in contumacia. Mina ha appena il tempo di interrogarsi sul significato della parola "contumacia" che i quattro le saltano addosso. Inizia quello con le basette, poi, uno a uno, le danno quella che nella sua autobiografia in musica racconta come una «solenne ripassata»..$^{2}$ L'unica figura presente in casa oltre a Mina è la nonna, paralitica e con le cataratte, che di lì a poco si sentirà male e cadrà in uno strano assopimento. La scrittura si muove continuamente tra il detto e il non detto, e per la narrazione dello stupro, per quanto scarna ed essenziale, dovremo aspettare di leggere La spirale della tigre. La bambina cerca di nascondere come può i segni immediatamente visibili, ripulendo ossessivamente se stessa e il pavimento dal sangue e dall' «orina» degli uomini; smetterà solo al ritorno della madre e della sorella maggiore. La quiete illusoria si spezza dopo pochi istanti: l'emorragia causata dalla violenza si riapre, e il sangue che la bimba aveva pulito con tanta cura riprende a scorrere. Mina non cerca nemmeno di far comprendere alla madre che il sangue non è dovuto al menarca ma alla violenza subita: sa che scoprire la verità celata nel ventre della figlia sarebbe stato per la madre un 
dolore troppo grande. La bambina vive però le conseguenze del trauma, e, per quanto lo voglia nascondere alla madre, ha bisogno d'aiuto. Desidera, forse inconsciamente, che la madre sollevi il telo nero che è calato a separarle, che veda e interpreti il silenzio della figlia, senza fare domande. La madre a tratti sembra farsi più vicina, e le tre rughe fra le sopracciglia si trasformano nella schin (שU), la lettera dell'alfabeto ebraico che rappresenta la maturità della donna. La schin unisce madre e figlia, e alimenta l'urgenza di confidarsi della bambina:

la schin di mia madre si scalda. Potrebbe diventare la mia schin e non sarei più morta. Troppo tardi: dovrebbe vedere senza chiedere [...] (LIMENTANI 2013, 32).

La possibilità di salvezza offerta dalla madre svanisce in fretta: la schin si disfa e smette di tentarla «con le dolcezze di un calore pericoloso» (ivi, 23).

Il continuo oscillare della narrazione tra presente e passato, tra realtà e incubo e tra luoghi diversi, ci porta d'improvviso in una casa di fronte al mare, poi nel paese di Cave, poi ancora nel convento dove Mina viene ospitata assieme alla sorella, e infine per le strade di Roma il giorno della Liberazione e nell'atrio del Tempio, che si fa testimone degli orrori della Shoah, ospitando i presenti ma soprattutto gli assenti. È una trama a stento percepibile, che mescola diverse scene e momenti dell'esistenza. La narrazione procede per frammenti, senza alcuna linearità. Impossibile ritornare a quei momenti e raccontarli: quando l'incomunicabilità si infrange sono le emozioni scaturite dal trauma, la memoria profonda e il corpo di Mina a trovare rappresentazione.

Le prime pagine di In contumacia, quelle in cui la scrittura si sofferma sull'interno domestico in cui si è consumata la violenza, sono caratterizzate dall'instabilità di una scrittura che testimonia la portata emotiva del trauma. Difficile comprendere dove si situi la verità; sono gli attimi in cui Mina si trova ad affrontare un dolore per lei inedito, che le squarcia ventre e anima: in parte ne comprende le ragioni, in parte le restano oscure. «Chi è vivo e chi è morto?» è una delle tante confuse domande che Mina si pone in quei momenti. Morti sono i quattro fascisti che le hanno dato la "ripassata», ma a essere morta è anche lei stessa. È morto chi sa, ed è per questo che la bimba deve nascondere a tutti quello 
che è successo. «Una cosa non esiste se nessuno la sa» (ivi, 18), e tenerla celata è l'unico modo per non contaminare e di conseguenza non uccidere chi le sta vicino. Al contrario, è vivo chi non sa. È viva la nonna, ma soprattutto sono vivi i suoi occhi malati, che non possono vedere i quattro uomini addosso alla nipote. Anche il dolore che lacera i fianchi di Mina è vivo. Per tutto il romanzo la bimba non capisce se sia puro o impuro, dovuto a una «fabbrica» che qualcuno le ha messo nel ventre senza chiederle il permesso, o a una innocua colica mestruale, come è convinta la madre. Mina non comprende il significato del suo dolore, che perciò resta vivo. Vivo, infine, è anche l'altro grande spettatore di queste scene, il gatto, che con silenziosa agilità felina si mantiene al riparo dalla contaminazione. La memoria invece è viva quando è «abitata da fantasmi, ombre, immaginazione», quando è «avvolta nella nebbia» (Goldkorn 2016, 63), scrive Wlodek Goldkorn ne Il bambino nella neve, lettura che la stessa Giacoma Limentani mi consigliò, e che molto del suo rapporto con la memoria mi ha aiutata a comprendere.

Avvolto nella nebbia, il gatto diventa una palla bianca, poi il pappo di un fiore che si disperde nell'aria e trasporta pulviscoli di luce, ma nel contempo si scinde in quattro squadristi. Questi, a loro volta, si uniscono per diventare una sola violenza, il grumo di ferro che lacera il ventre della bambina. E il grumo è un nastro, che si distende come i Sefarim, i rotoli su cui viene trascritta la Torah. Il grumo diventa poi l'uomo con la basette, o addirittura una fabbrica che produce l'uomo con la basette, in una sorta di continua riproduzione della violenza.

Si trasforma anche la casa in cui si è consumata la violenza, l'ambiente domestico che avrebbe dovuto salvaguardare la bambina ma nulla ha potuto fare contro l'invasore, e che sembra ora partecipare empaticamente allo spaesamento e al dolore di Mina. A cominciare dalla lama di pulviscolo, che ora cala sulle circostanze e confonde le immagini, ora si sposta, ora dimentica. Il tavolo, sotto il quale Mina trova rifugio, sembra trasformarsi in una casa, per offrire alla bambina la protezione che quella vera le ha negato. I biscotti portati a casa dalla madre, infine, vengono versati nella biscottiera facendo il rumore delle «cose che 
si lacerano in segreto» (ivi, 27), come Mina e il suo ventre. Ventre che piange per lei, dal momento che la bimba ride e vomita ma non riesce a versare lacrime.

Molti altri liquidi, corporei ma non solo, iniziano a scorrere alla seconda pagina del romanzo e lo percorrono fino alla fine. Non appena i fascisti lasciano la casa Mina comincia a pulire i residui lasciati sul pavimenti da loro e da se stessa, spaesata dai liquidi maschili. L'«orina» degli uomini rappresenterà un vero e proprio incubo per la bambina e per la donna che diventerà. Mina la riconosce nella chiara d'uovo che il dottore le consiglia di prepararsi come cura ricostituente, e nell'olio canforato inserito nella siringa per la nonna. Di quest'olio si riempirà poi, nel sonno, il suo letto di donna ormai adulta e sposata.

L'altro flusso che attraversa il romanzo è quello del sangue, buono e cattivo, puro e impuro. È visto da Mina come una condanna, un'imposizione, una vergogna a cui vorrebbe rinunciare. Infetterà chiunque lo tocchi: è il sangue dell'emorragia causatole dagli squadristi, e la renderà «intoccabile» ogni mese. Come sottolinea Lucamante,

fluidi corporei che formano parte ancora oggi della fierezza di essere donna improvvisamente divengono liquidi luttuosi, una vischiosità di cui vergognarsi. [...] Fatti normali come il mestruo vengono contrapposti alla violenza giustificata del Fascismo (LUCAMANTE 2012, 254-260).

E cosa meglio del liquido amniotico - ancora un altro liquido - può proteggere e nascondere questa vergogna, di cui la madre sembra essere molto fiera? Mina trova rifugio nella camera da letto della madre, trasformata in una placenta, e lì nasconde il suo segreto. Interviene la madre a rassicurarla: questo «ciclo delle maree», se proprio non lo vuole, si interromperà con la prima gravidanza. Ma Mina non le crede, non riesce a credere più a nulla di quanto le viene detto. $\mathrm{E}$ infatti nessuna gravidanza arriverà mai a interrompere queste maree, il nastro impuro continuerà a srotolarsi.

Le immagini che scorrono tra le pagine di In contumacia sono di difficile comprensione, sia per la bambina protagonista che per il lettore e la lettrice: come i fantasmi di Goldkorn, su di esse calano di continuo un fastidioso 
pulviscolo, un telo nero, e le cataratte della nonna, che, come nota Lucamante, sembrano espandersi a tutto il mondo di Mina, confondendolo.

Se esiste un pulviscolo che impedisce di vedere, ve ne sono altri che impediscono di capire. Primo fra tutti, il linguaggio degli adulti. La domanda della bambina circa il significato del termine "contumacia" resta inascoltata fino alla fine del libro: Mina tenta allora di trovarsi da sola la risposta, lasciando risuonare la parola e dandole diversi significati di volta in volta. Il linguaggio ostacola anche il racconto della «ripassata» dei quattro bravi: oltre alla mancata consapevolezza della violenza sessuale subita, la bambina non conosce le parole per raccontarla alla madre, ed è questa la ragione del suo silenzio, che si fa «unica possibile comunicazione del dolore» (ivi, 53).

Oltre al pulviscolo che confonde vista e linguaggio, allo sfocamento delle immagini contribuiscono l'età infantile e l'innocenza di Mina, che troppo poco ha vissuto per poter essere consapevole di ciò che le è accaduto. Sa che il suo corpo sta lasciando l'infanzia per entrare nella pubertà, e che agli uomini non possono fare quello che hanno fatto a lei, ma le sue domande sono più confuse che mai:

Io sono una signora o una signorina? Se sono una signora ho quattro mariti. [...] A dodici anni si sta seduti composti. Lo dice la nonna e anche la mamma. Chissà perché? Forse perché a dodici anni cresce il petto. Ma il petto non ha niente a che vedere con le gambe. Però quello lo morde mentre l'altro tiene le gambe. L'altro ancora tiene le braccia e quello con le basette mette il dolore che adesso apre i fianchi (ivi, 19).

La nonna, l'unica a poterle dire se il suo ventre si sia trasformato in una «fabbrica», sembra destinata a non risvegliarsi più, e la domanda di Mina potrebbe restare per sempre sospesa nel vuoto. Troverà per fortuna risposta, ma non in questo romanzo: bisognerà prima aspettare di possedere la lingua che permette di «descrivere il silenzio» (LUCAMANTE 2012, 251).

\section{Il Dono della memoria familiare}

La lingua che la guida verso una più lieta comprensione del mondo è quella trasmessale dal padre Leonello e dal nonno Beniamino, e Dentro la $D$ ne è 
l'alfabeto. La D ha molteplici significati, ma è sottolineandone il valore numerico che viene introdotta e descritta: «Eccola la Dàlet. Quarta lettera dell'alfabeto ebraico e numero quattro. Quattro come le lettere del Tetragramma [...]» (LIMENTANI 2013, 114).

Come non ripensare ai quattro fascisti di In contumacia? La Dàlet sembra ricordarci che di quella violenza si sente ancora un'eco nemmeno troppo lontana, ma che si sta anche provando ad aprire una porta verso la dignità. Non solo la Dàlet ha la forma di una porta aperta, ma può anche leggersi Dèlet, che significa proprio porta. Se nel primo romanzo dominavano il trauma e la non comprensione, in Dentro la $D$ il pulviscolo lascia spazio a una ricomposizione con l'infanzia, che avviene grazie al recupero dell'identità ebraica. Nella Dàlet si riassumono tutte le lettere che formano il nome di Giacoma Limentani: il recupero del proprio passato e della genealogia familiare si fanno Dono3 e permettono di prospettare una speranza futura.

Comincia dunque la sua indagine relativa a vicende legate alla sua tradizione familiare: mendica i ricordi di cui non può avere consapevolezza per costruire storie parallele. L'ossessività è quella dell'archivista, lo schedario quello del cuore. Presente e passato, suoi e degli altri, si fondono assieme in un unico grande mosaico. Le storie raccontate sono quelle di tutti gli ebrei romani, nessuno escluso: quelli incontrati e quelli che avrebbe invece voluto conoscere, se non fossero vissuti in luoghi e tempi da lei lontani. Capita che lo stesso personaggio si trovi, un po' cambiato, nei tre libri; a volte anche in storie diverse all'interno dello stesso romanzo. Rimangono i fondamenti della vicenda, ma possono cambiare il suo nome, tratti del suo carattere e della sua storia, persino il rapporto di parentela che intrattiene con colei che narra la storia. Questa continua rielaborazione delle vicende dei personaggi le viene in parte dalla modalità di interpretazione dei testi sacri ebraici nota come midrash, in parte dalla volontà di narrare tutte le possibili sfumature di un personaggio, quelle omologate dalla memoria ufficiale della Shoah. È una scrittura tendente a un continuo nomadismo, e nomadi sono anche i personaggi. Nemmeno l'indagine 
compiuta segue un andamento lineare, ma proprio in questo sta la sua più grande ricchezza e vivacità. Iniziando una digressione che potrebbe sembrare allontanarla «dal fulcro dell’inchiesta», prosegue così:

Se poi l'allontanamento sarà reale, pazienza: in una caccia al tesoro, anche i depistaggi fanno parte del gioco, e non è detto che in fondo al cuore io non desideri proprio allontanarmi dal tesoro che dichiaro di andar cercando (ivi, 139).

Struttura portante di Dentro la $D$ è il gioco linguistico, che non tralascia le finali ma si focalizza soprattutto sulle iniziali di parola: una diretta eredità del nonno Beniamino, il quale le iniziali «alla maniera di un caleidoscopio le faceva rifrangere in mille immagini cangianti» (ivi, 112). Dei suoi libri, a Giacometta, piace pensarsi l'erede. La passione per le iniziali deriva ancora una volta dal gioco midrashico, che non opera solo sul testo, ma su singole lettere. È per questa attenzione alle iniziali di parola che il padre Leonello viene in questo romanzo chiamato Davìd. Davìd significa l'amato e inizia con la Dàlet, che rappresenta il nutrimento (dover, pastura), ed è intimamente legata alla Dèlet, la porta. Il padre è stato colui che le ha aperto la porta verso la dignità, che i romanzi di Giacoma Limentani si fanno poi carico di trasmettere a chi legge.

Il romanzo si apre proprio sul padre, «vulnerabile al pianto dei neonati» (ivi, 99), che fatica a comprendere la fiducia delle madri nel potere calmante del loro seno, dal momento che, dopo la poppata, i bambini stanno quieti per un tempo troppo breve. Il latte maschile, in Dentro la $D$, sembra essere casher quanto quello femminile, e forse anche più di questo. Il riferimento sotteso è al Libro di Ester, in cui Mardocheo deve nutrire la nipote orfana a lui affidata, e, non trovando una balia che voglia allattarla, se la appoggia al petto e aspetta che Ester faccia uscire del latte «dalle sue povere ghiandole maschili e atrofizzate» (LIMENTANI 1997, 33).

Il padre David compie in Russia un gesto simile, trovando in una stazione affollata una bimba perduta dalla madre, e calmando i suoi singhiozzi facendole bere una bottiglia di latte recuperata da una contadina con una mucca. La piccola Anja e la madre Dar'ja, poi ritrovata, seguono Davìd a Roma, e le due restano per 
alcuni anni amiche di famiglia. Anja e Mina crescono legate dal comune «Destino di venire allattate da estranei» (LIMENTANI 2013, 117), dal momento che anche la madre di Mina deve cercare una nutrice per la figlia. Quando il padre comprende che Mina invidia la perfetta bellezza di Anja, la porta alla galleria Borghese e poi a quella d'arte moderna, per mostrarle il Narciso di Caravaggio e la Donna in bianco di van Dongen: la sposa simile a un giglio del primo quadro rappresentava per Davìd una perfezione non desiderabile, mentre la donna del secondo era la vera realtà femminile.

L'arte la avvicina al padre anche quando lui se ne è andato ormai da qualche anno. Nel corso di un viaggio ad Amsterdam, al Museo Stedelijk si trova a osservare due quadri di Daniel Spoerri, uno raffigurante una valigetta e l'altro un «letto sconvolto dall'insonnia con sopra, fra la riversina gualcita e il cuscino devastato, gli avanzi di un pasto incongruo» (ivi, 116). Il primo la riporta al padre, che dentro l'armadio era solito tenere una valigetta pronta per qualsiasi eventuale partenza, e il secondo la rimanda invece al letto su cui aveva dormito lei la notte prima. Il titolo del primo, The insane assaulter, e il fatto che in entrambi i quadri, nello stesso punto, ci fosse una bottiglia con il tappo a molla, la porta a chiedersi se anche in suo padre, come in lei, «non ci sia stata una qualche vena di insania» (ivi, 117).

«Nella vita e nell'arte non si sa mai dove sia il tempo: spesso è la fine a prevedere l'inizio» (ivi, 178), le aveva insegnato qualche giorno prima di morire, davanti alla Ragazza in piedi di Schiele: il contrario di ciò che accade al Destino delle parole. Al Destino delle iniziali si affida anche la madre Gemma, che ha vissuto abbastanza a lungo accanto alla famiglia di Davìd per capire che la balia di Mina deve essere scelta anche sulla base del nome: Daria. È però la madre, innalzato il telo nero che nel primo romanzo la separava dalla figlia, a occuparsi del nutrimento dell'anima di Mina, inondandola di canzoni anziché di latte. Proprio dalla musica viene uno dei suoi più grandi insegnamenti, che ricorda in parte l'andamento dell'interpretazione midrashica arrivatale per via paterna: 
Mi dimostravi quanto sia errato imprigionare certi fraseggi in rigidi schematismi contrappuntistici che soffocano il canto del tema. Mi davi il tema e mi mostravi quante variazioni potevano scaturirne (ivi, 169).

\section{Spirali di memorie}

La spirale della tigre contiene le risposte alle domande che erano rimaste inevase nel primo romanzo, e offre un risarcimento simbolico alla violenza subita dalla bambina protagonista. Del trauma, però, si parlerà soltanto nelle ultime pagine: gran parte del romanzo è un intreccio di storie che sfiorano la vita di Mina ma non la riguardano direttamente. Il nucleo centrale è il racconto della componente ebraica di Roma durante il ventennio fascista, la stessa di Dentro la D, ma molteplici trame la intersecano e ci portano in luoghi e tempi lontani: nell'Avignone papale, a Livorno, e per le stradine del ghetto di Roma prima e dopo la sua apertura da parte delle truppe piemontesi. Una rete di sguardi, da quelli scambiati tra Leone Scarnicchia e la tigre impagliata, a quello tra la protagonista e il suo stupratore, collega i diversi racconti. I ricordi di Giacometta, che riportano in vita queste storie, sono come i rammendi della nonna Elena, «ricami che fissano trame connettive sulle lacerazioni dei tessuti» (ivi, 269).

Il romanzo si apre con la storia del trisavolo Leone detto Scarnicchia, «l'uomo più mite del ghetto di Roma» (ivi, 186) e venditore ambulante. Per arrotondare gli scarni guadagni, inizia a contrabbandare brocche d'acqua miracolosa. Il successo è tale che Leone viene fermato persino da un gruppo di suore, le quali, desiderando ardentemente l'acqua magica dell'uomo, lo portano nel loro convento. Qui, gli occhi color ambra di una tigre impagliata attirano l'attenzione di Leone, che in cambio dell'acqua chiede di portarsela a casa.

«La tigre era ferma in un angolo, pronta a balzare, e lo guardava» (ivi, 189). Potrebbe balzare in qualsiasi momento, su di lui, su Giacometta, e su tutti gli altri personaggi di questo romanzo, dei quali Leone vede forse il riflesso negli occhi ambrati dell'animale. Potrebbe balzare dentro e fuori dal tempo: «Impossibile parlarne al passato. $\mathrm{Ci}$ sono cose che gravitano in un continuo presente» (ivi, 
268). Sono le parole che seguono la reazione di Giacometta all'incontro con il suo stupratore, e aiutano a interpretare non solo La spirale della tigre ma l'intera trilogia. La storia umana gravita in un istante presente e anticipa il tempo futuro, ed è per questo che si fanno fondamentali i ricordi e la memoria. È un tempo che segue un andamento non rettilineo, un divenire raramente conclusivo.

Da qui scaturisce anche la sua scrittura: come le settanta striature che avvolgono in una spirale il corpo della tigre impagliata, allo stesso modo procedono le narrazioni di Giacoma Limentani, frutto di una memoria che si sviluppa su piani diversi. Le risulta impossibile mettere in ordine gli avvenimenti che hanno segnato la sua vita, tralasciando quelli che l'hanno solo sfiorata da lontano come le consiglia di fare la madre, poiché proprio questi eventi hanno frantumato il suo tempo per poi riunirlo in un mosaico che ne comprende tanti altri. È per questo che i suoi romanzi sono ben lontani dall'avere un andamento diaristico, che per Limentani è un «rituale che segue il fluire del tempo». Al contrario, scrive,

le memorie mi stanno dentro a grappoli di ricordi, e i ricordi mi costringono a un continuo nomadismo fra il mio tempo e i tempi e luoghi delle persone che ho amato, come pure di quelle che avrei potuto amare se le avessi conosciute [...] (ivi, 198).

Le spirali di memorie intrecciano narrazioni gioiose a quelle della crescente discriminazione ebraica a Roma: La spirale della tigre è anche il romanzo in cui ritornano il trauma e la violenza fascista. Vista dagli occhi di una bambina, viene raccontata soprattutto in relazione alle istituzioni scolastiche. Se ne era già accennato in Dentro la $D$, quando Mina e le amiche Donata e Carla iniziano a chiedersi se si potesse ancora chiamare patria un paese in cui le scuole stavano chiudendo loro le porte in faccia. Ne La spirale della tigre i ragazzi ebrei vengono strappati alle loro scuole per essere reinseriti in un unico edificio solo a loro destinato. Tutti insieme, quelli più ortodossi e quelli nemmeno praticanti, e ognuno di loro è costretto a porsi domande sulla propria identità ebraica. Il mondo deformato dal fascismo emerge in tutta la sua brutalità nelle pagine in cui Limentani torna a raccontare la violenza subita - o meglio, la racconta 
davvero per la prima volta - e in quelle in cui affronta il suo stupratore. Lo riconosce subito, nonostante le basette siano state rasate. Giacometta si trasforma dapprima in un segugio, attendendo che si accorga di lei: le basta uno scambio di sguardi per recuperare le dignità. Lo segue per diversi giorni, fino a quando il figlio dell'uomo, Enzo, la riconosce come sua vecchia compagna di scuola al Mamiani. Decide di presentarla al padre, questa ebrea che fortunatamente era nata in Italia; e Mina, dopo tre romanzi, rompe finalmente il silenzio:

«Mi dispiace dovertelo dire, ma non tutti gli italiani sono stati brava gente, e non tutti i fascisti hanno tenuto i pugni in tasca. Forse tuo padre non frequentava assiduamente il fascio di questo quartiere, altrimenti saprebbe che lì si manganellava sodo». Cerco di incontrare occhi che vorrebbero sfuggirmi mentre dico: "Chiedilo a Livio se lo vedi ancora, caro il mio Enzo. Ti dirà che suo padre e il mio ne portano ancora le cicatrici. E da quel fascio partivano squadracce che si divertivano a violentare la bambina che ero, e che ti piaceva tanto. [...] Vomito lì, per la strada, con entrambe le mani premute contro la parete della casa dove lui abita. Vomito l'uomo che mi è entrato nella carne, mentre quello cui ispiro terrore mi si stabilizza nel cervello. Vomito davanti a un figlio che non sa più cosa pensare del proprio padre (ivi, 268).

Vomita tutto quello che avrebbe voluto dire il giorno della «ripassata» e per tutti gli anni seguenti, e le domande che non hanno mai trovato risposta. Vomita il suo silenzio e il suo atto di eroismo, ed è l'ultimo liquido a scorrere tra le sue pagine, richiudendo simbolicamente le ferite da cui i flussi di In contumacia sono continuati a uscire per lungo tempo. Affinché il passato smetta di proiettare ombre sul futuro e il ricordo possa farsi dono e speranza di guarigione, occorre sempre scrivere dopo. Le memorie dolorose dell'infanzia vengono recuperate nel primo romanzo, ma la distanza temporale dai fatti trattati è ancora troppo breve perché la scrittura possa farsi narrazione. Le pagine iniziali de La spirale della tigre sembrano essere chiave di lettura dell'intera Trilogia, nel momento in cui il trisavolo Leone si specchia negli occhi della tigre impagliata:

Affascinato, Leone si avvicinò per specchiarsi meglio. Gli occhi di vetro si fissarono nei suoi, vuoti d'immagini. Qualcosa, forse un tarlo nell'impagliatura, scricchiolò. Leone fece un salto indietro e rivide l'ebreo e tutta la stupenda bestia nell'insieme (ivi, 189). 
Come Leone deve allontanarsi dalla tigre per poter vedere la propria immagine riflessa, così Limentani deve aumentare la distanza tra il tempo della scrittura e quello del trauma: solo allora, dopo aver storicizzato il dolore, sarà possibile passare dal flusso di coscienza di In contumacia agli intrecci di narrazioni de $L a$ spirale della tigre.

Riattraversare la memoria e scrivere dopo non determina un divenire lineare, e La spirale della tigre non è il romanzo conclusivo della Trilogia: il midrash insegna a non smettere di cercare la verità, a domandare, senza accontentarsi delle risposte che si trovano di volta in volta. Nei confronti del proprio passato e presente l'io si trasforma continuamente, si trasformano i personaggi e le loro relazioni con colei che narra, si trasforma soprattutto la memoria, che a tratti paralizza e a tratti diventa dono.

La scrittura letteraria di Giacoma Limentani infrange qualsiasi ordine, consapevole che lo stare tra realtà diverse e a volte conflittuali è una ricchezza. In riferimento alle molteplici lingue che fin dalla prima infanzia è chiamata a padroneggiare, definisce «contrasto» il rapporto con la necessità di tradursi:

Il contrasto insito nella stessa parola contrasto, che posso dividere in con-tra-sto. E cioè vivere con la consapevolezza di stare sempre tra realtà e lingue diverse, che non possono non essere una ricchezza (LIMENTANI 2004a, 141-142).

Sono contrasti anche il continuo gravitare fra tempi diversi delle sue narrazioni, e il suo nomadismo tra la letteratura e l'ebraistica, senza riuscire a scegliere a quale gruppo appartenere: gli scrittori la considerano una studiosa di ebraismo, gli ebraisti una scrittrice. È felice al convegno della Società Italiana delle Letterate del 2002, finalmente invitata da letterate a parlare di letteratura, ma invita a ricordare che la ricchezza dell'arte sta negli sconfinamenti tra etichette. Ne risulta un mosaico di generi letterari, le cui frontiere sono attraversate in continuazione: la narrativa e la saggistica; l'ebraistica, nelle sue declinazioni alte del midrash e della qabbalah, nella tradizione popolare propria dell'area romana, nelle leggende della tradizione Talmudica che il padre le racconta per farla addormentare, e infine nel chassidismo di Nachman di Breslav, che durante 
l'inferno della guerra si fa «sogno» consolatorio. E poi ancora la fiaba: conclude Dentro la $D$ e ritorna nelle pagine finali de La spirale della tigre, quasi a rappresentare un ultimo momento di riflessione su ciò che si è appena narrato, che acquisisce così una nuova luce e tocca nel profondo tutte e tutti, facendosi ponte verso la comunicazione e la relazione. È poi l'intera Trilogia ad avere l'andamento della fiaba, e in modo particolare i racconti a spirale dei personaggi della tribù del ghetto che si snodano in Dentro la $D$ e ne La spirale della tigre. Realtà e finzione si mescolano di continuo, impedendo la mera trascrizione dell'esperienza reale. I nomi dei personaggi, i rapporti di parentela, e alcuni tratti del loro carattere e della loro storia cambiano di romanzo in romanzo. Il procedere per fiabe e leggende le viene ancora una volta dalla tradizione ebraica: trasformare la realtà in «fiabesca leggenda», si chiede in un intervento su Etty Hillesum, «non è esattamente quello che i Maestri ebrei hanno sempre fatto» (LimENTANI 1997, 131)? Il midrash non è una rielaborazione creativa di passi biblici, tesa a illuminare un particolare o un personaggio della Scrittura? E di nuovo il chassidismo: anche Nachman di Breslav riteneva che la sapienza del mondo si manifestasse in canto fiabesco.

Se per Etty Hillesum l'unico modo per raccontare il campo di Westerbork era usare il linguaggio delle fiabe, per Giacoma Limentani raccontare il trauma quando questo sembra incomunicabile significa tradurre dai luoghi più remoti dell'anima. Anche quando ciò che si scrive «avrebbe voluto avere l'asettico distacco di un saggio scientifico», perché qualunque cosa si scriva rivela quel che l'autrice è:

Più passa il tempo e più scrivo, [...] e più rileggo quanto ho scritto nell'assoluta convinzione e volontà di scrivere storie di fantasia, saggi di commento a passi biblici e perfino filastrocche per bambini, più mi accorgo di scrivere sempre me stessa e tutto quanto mi ha fatta come sono. Una Babele di nascondimenti e rivelazioni, lingue materne e traduzioni, bisogno di aprirmi e ostacoli verbali che, se superati, dopo pagine e pagine strappate mi portano a quell'unica pagina che spero di poter definire letteraria. Che lo sia davvero non sta a me giudicare. Né potrei: sono troppo coinvolta (LIMENTANI 2004a, 143). 


\section{Memoria collettiva}

Non è solo con se stessa e con la scrittura che Giacoma Limentani intesse una relazione: la memoria necessita dell'altra e altro da sé, per trovare ascolto e per trarne nutrimento reciproco. Dal suo viaggio alle radici del sé Limentani trascende di continuo, intrecciandolo a racconti su personaggi della tribù del ghetto, di Livorno e di Avignone. Restituendoli alla vita attraverso la pagina scritta, disegnandone mappe e alberi genealogici, si fa carico di tutti loro, narrando la particolarità che è in ogni loro vita. Anche quando è privata, la memoria diventa collettiva.

È necessario però chiedersi che cosa significhi una memoria collettiva della Shoah. Ascoltando degli studenti chiamati a commentare un film tratto da $L a$ tregua di Primo Levi, resta colpita dalle loro conclusioni. La tregua concordavano i ragazzi e con loro gli insegnanti - racconta un ritorno; una storia che, pur partendo da un luogo atroce, conosce un finale lieto. A prescindere dalle storie e dalle memorie personali antecedenti la discriminazione e la deportazione, i ritorni da Auschwitz sembrano essere, in questo livellamento dei ricordi, uguali e gioiosi per tutti. Affinché la memoria possa essere foriera di speranza, occorre invece distinguere quella davvero collettiva da quella «storicamente inoppugnabile» (LIMENTANI 1998, 126) che si arroga il diritto di omologare i ritorni, nonostante quei ritorni siano «troppo legati a memorie precedenti ad Auschwitz» (ivi, 127)

Di Shoah e di cultura ebraica, scrive Limentani, si è parlato in maniera incompleta e fuorviante, seguendo mode ed etichette, ricostruendo «senza spazzar via le macerie» e mettendo una «pietra sul passato» (LIMENTANI, 1997, 11-10). Si intitola proprio Tempi mode etichette e sentimento il suo intervento a un convegno sullo scrivere dopo Auschwitz connesso all'identità ebraica, uno dei tanti momenti in cui Limentani riflette sulle difficoltà della testimonianza. A volte, dinnanzi a un pubblico che, in parte per indifferenza e in parte per la paura di soffrire il dolore altrui lasciandosi coinvolgere, sembra non conoscere l’imperativo ebraico all'ascolto, la testimonianza può farsi talmente dolorosa da 
preferirle il silenzio. Le memorie non condivise alzano un confine invisibile tra i perseguitati e gli altri. Riprendendo Les cadres de la mémoire di Maurice Halbwachs, chi ricorda tragedie che gli altri non possono comprendere somiglia a chi vede quello che altri non vedono: sono entrambi degli allucinati, degli esiliati. Personaggi scomodi, che infastidiscono, «specie se le allucinazioni di cui soffrono sono tali da sollecitare sensi di colpa nella società stessa cui desiderano comunicarle» (LIMENTANI 1998, 126). L'allucinato può scegliere il silenzio oppure comprendere che il termine ebraico per esilio, galut, proviene dalla stessa radice di galah, che significa invece illuminare, come a dire che «l'esperienza dell'esilio illumina» (LimENTANI 1997, 15).

Ma non può essere Auschwitz a illuminare: le memorie che gravitano nei romanzi di Giacoma Limentani sono quelle che dalla Shoah sono state infrante. Riprendo ancora le parole di Goldkorn:

"Mai più Auschwitz". Per me sono parole prive di senso e contenuto: Auschwitz c'è stata, la Shoah c'è stata, dire "mai più" o "non permettere che si ripeta" significa costruire una specie di pedagogia della Shoah, cercare di trarre una lezione dalla catastrofe. [...] La Shoah è solo un vuoto. [...] Negare che il vuoto è vuoto, cercare di riempirlo con presunti significati positivi e con un messaggio di speranza è peggio dell'angoscia: è il rifiuto di capire quanto il Male sia radicato dentro ognuno di noi (GOLDKORN, 2016, 132-133).

La speranza va ricercata prima del vuoto, nell'amore e nella nostalgia per ciò che di buono è andato perduto. Prendendo le mosse dai «comportamenti dell’ieri» (Limentani, 2006, 53) per «costruire un sempre più degno domani». Scrivendo dopo per scrivere prima: «prima che la speranza si avveri», per «cercare almeno di prospettarla» (LIMENTANI 1997, 189). 4 
Note

${ }^{1} \mathrm{Si}$ vedano ad esempio Marlene Heineman, Gender and destiny: women writers and the Holocaust, Connecticut, Westport, 1986, e Carol Rittner - John K. Roth, Women and the Holocaust. Different Voices, St. Paul, Minnesota, Paragon House, 1993. In ambito italiano si vedano Lucio Monaco (a cura di), La deportazione femminile nei lager nazisti, Milano, Franco Angeli, 1995; Roberta Ascarelli (a cura di), Oltre la persecuzione. Donne ebraismo memoria, Roma, Carocci, 2004; Giovanna De Angelis, Le donne e la Shoah, Roma, Avagliana Editore, 2007; Alessandra Chiappano (a cura di), Essere donne nei Lager, Firenze, La Giuntina, 2009.

${ }_{2}^{2}$ Una traduzione del vissuto attraverso canzoni che hanno segnato istanti e fasi della sua vita, rappresentando una valvola di sfogo rispetto alla drammaticità dell'esistenza. Più che le canzoni stesse, che proseguono poi con le proprie storie, sono i titoli a diventare per lei un vero e proprio lessico famigliare.

3 Si riprende l'immagine della memoria come Dono da un contributo di Alessandra Pigliaru per «Leggendaria» 102 (novembre 2013), Il destino di ogni parola è nell'inizio.

4 Le conclusioni di questo contributo, in particolare le riflessioni sulla memoria del bene perduto, sono confluite in Chiara Xausa, Il poco che sorregge il molto, in Adriana Chemello (a cura di), Il mosaico della memoria. Omaggio a Giacoma Limentani, Roma, Iacobelli, 2017.

\section{Bibliografia}

Opere di Giacoma Limentani:

LIMENTANI 1967

Giacoma Limentani, In contumacia, Milano, Adelphi, 1967.

LIMENTANI 1992

Giacoma Limentani, Dentro la D, Genova, Marietti, 1992.

LIMENTANI 1993

Giacoma LiMENTANI, Nachman racconta, Firenze, Giuntina, 1993.

LIMENTANI 1996

Giacoma LimENTANI, Il Midrash: come i Maestri ebrei leggevano e vivevano la Bibbia, Milano, Paoline, 1996.

LIMENTANI 1997

Giacoma LimentanI, Scrivere dopo per scrivere prima, Firenze, Giuntina, 1997.

LIMENTANI 1998

Giacoma LiMENTANI, Far rivivere il bene perduto, in La Sho'ah tra interpretazione e memoria, Atti del Convegno Internazionale di Napoli, 5-9 maggio 1997, Napoli, Vivarium, 1998, pp. 125-133.

LIMENTANI 2001

Giacoma Limentani, Regina o concubina? Ester, Milano, Paoline, 2001.

LIMENTANI 2003

Giacoma LimENTANI, La spirale della tigre, Varese, Giano, 2003. 
LIMENTANI 2004a

Giacoma Limentani, Tra due P: Pensiero e Penna, in Lo spazio della scrittura.

Letterature comparate al femminile, a cura di Tiziana Agostini et al., Padova, Il Poligrafo, 2004, pp. 131-143.

\section{LIMENTANI 2004b}

Giacoma Limentani, Donna fra donne, in Oltre la persecuzione. Donne, ebraismo, memoria, a cura di Roberta Ascarelli, Roma, Carrocci, 2004, pp. 93-94.

\section{LIMENTANI 2006}

Giacoma LIMENTANI, Aforisma: un poco che sorregge il molto?, in La brevità felice. Contributi alla teoria e alla storia dell'aforisma, a cura di Mario Andrea Rigoni, Venezia, Marsilio 2006, pp. 51-61.

LIMENTANI 2008

Interventi alla tavola rotonda del 7 giugno 2007, in Memoria collettiva e memoria privata: il ricordo della Shoah come politica sociale, Atti del Convegno di Roma, 6-7 giugno 2007, a cura di S. Lucamante et al., Utrecht, Universiteit Utrecht, 2008, pp. 219-229.

LIMENTANI 2013:

Giacoma LimENTANI, Trilogia, Roma, Iacobelli, 2013.

\section{Bibliografia critica relativa a Giacoma Limentani:}

CHEMELLO 2017

Adriana ChEMELlo (a cura di), Il mosaico della memoria. Omaggio a Giacoma Limentani, Roma, Iacobelli, 2017.

Di CORI, PONTECORVO 2002

Paola Di CORI, Clotilde PONTECORvo (a cura di), Parole e silenzi: scritti per Giacoma Limentani, Torino, Trauben, 2002.

CARÙ 2004

Paola CARÙ, Raccontando le radici: la riscrittura di sé e della storia nei romanzi di Giacoma Limentani, in Lo spazio della scrittura. Letterature comparate al femminile, a cura di Tiziana Agostini et al., Padova, Il Poligrafo, 2004, pp. 225-231.

LUCAMANTE 2012

Stefania LuCAmante, Dentro la $D$ e fuori dal ghetto con le bambine di Roma: Lia Levi, Rosetta Loy e Giacoma Limentani, in Stefania Lucamante, Quella difficile identità. Ebraismo e rappresentazioni letterarie della Shoah, Roma, Iacobelli, 2012, pp. 208-267.

LUCAMANTE 2013

Stefania LuCAMANTE, Postfazione, in Giacoma Limentani, Trilogia, Roma, Iacobelli, 2013,pp. 281-295.

Pigliaru 2013

Alessandra Pigliaru, Il destino di ogni parola è nellinizio, in «Leggendaria», XVII, 102 (2013), Novembre, pp. 32-34. 
Ulteriori testi consultati:

ASCARELLI 2004

Roberta ASCARElli (a cura di), Oltre la persecuzione. Donne ebraismo memoria, Roma, Carrocci, 2004.

BRAVO 1995

Anna BRAVO, Relazione introduttiva, in La deportazione femminile nei lager nazisti, a cura di Lucio Monaco, Milano, Franco Angeli, 1995, pp. 66-70.

BRAVO, JALLA 1986

Anna BRAVO e Daniele JALla (a cura di), La vita offesa. Storia e memoria dei Lager nazisti nei racconti di duecento sopravvissuti, Milano, Franco Angeli, 1986 (Milano, Franco Angeli, 2001).

BRAVO, JALLA 1994

Anna BRAVo e Daniele JALLA (a cura di), Una misura onesta. Gli scritti della deportazione dall'Ttalia 1944-1993, Milano, Franco Angeli, 1994.

CHIAPPANO 2009

Alessandra CHIAPPANO (a cura di), Essere donne nei Lager, Firenze, La Giuntina, 2009.

DE ANGELIS 2007

Giovanna DE ANGELIS, Le donne e la Shoah, Roma, Avagliana Editore, 2007.

FIORENTINO-TEDESCHI 1995

Giuliana FIORENTINO-TEDESCHI, Caratteri specifici della deportazione femminile, in La deportazione femminile nei lager nazisti, a cura di Lucio Monaco, Milano, Franco Angeli, 1995, pp. 28-30.

GOLDKORN 2006

Wlodek GOLDKORN, Il bambino nella neve, Milano, Feltrinelli, 2006.

HILLESUM 1990

Etty HILLESUM, Lettere (1942-1943), Milano, Adelphi, 1990.

LUCAMANTE 2008

Stefania LuCAMANTE, Fra storia vivente e memoria storica. Il ricordo della Shoah come politica sociale, in Memoria collettiva e memoria privata: il ricordo della Shoah come politica sociale, Atti del Convegno di Roma, 6-7 giugno 2007, a cura di S. Lucamante et al., Utrecht, Universiteit Utrecht, 2008, pp. I-XXVI.

LUCAMANTE 2012

Stefania LuCAMANTE, Quella difficile identità. Ebraismo e rappresentazioni letterarie della Shoah, Roma, Iacobelli, 2012.

MONACO 1995

Lucio Monaco (a cura di), La deportazione femminile nei lager nazisti, Milano, Franco Angeli, 1995 .

ROSSI-DORIA 1999 
Anna Rossi-Doria, Antisemitismo e antifemminismo nella cultura positivistica, in Nel nome della razza. Il razzismo nella storia d'Italia: 1970-1945, Atti del convegno di Bologna, 13-15 novembre 1997, a cura di Alberto Burgio, Bologna, Il Mulino, 1999, pp. 455-473.

SERENI 1993

Clara SERENI, Il gioco dei regni, Firenze, Giunti, 1993. 